\title{
A Study on the Benefit of TCP Packet Prioritisation
}

\author{
Eugen Dedu \\ Laboratoire d'Informatique \\ de l'Université de Franche-Comté (LIFC) \\ Montbéliard, France \\ Email: Eugen.Dedu@pu-pm.univ-fcomte.fr
}

\author{
Emmanuel Lochin \\ Université de Toulouse \\ ISAE - LAAS/CNRS \\ Toulouse, France \\ Email: emmanuel.lochin@isae.fr
}

\begin{abstract}
This paper studies and analyses the benefits of favouring the transfer of packets of a TCP flow over a besteffort network. Specifically, we aim at studying whether we could improve the pace of short data request, such as HTTP request, by giving a high priority to $\mathrm{TCP}$ packets that are not previously enqueued inside a core router. Following the idea that long-lived TCP flows greatly increase the routing queue delay, the motivation of this work is to minimise the impact in terms of delay, introduced by long-lived TCP flows over short TCP flows. Thus, this forwarding scheme avoids to delay packets that do not belong to a flow already enqueued inside a router in order to avoid delay penalty to short flow. We define metrics to study the behaviour of such forwarding scheme and run several experiments over a complex and realistic topology. The results obtained present interesting and unexpected property of this forwarding scheme where not only short TCP flows take benefit of such routing mechanism.
\end{abstract}

\section{INTRODUCTION}

Favouring the TCP connection establishment packets or any others packets belonging to a TCP flow inside a core network is not a novel idea. James Kurose, in his famous text book Computer Networking [1], suggests that it would be useful to protect from losses TCP packets with a low time-to-live value in order to prevent retransmission of packets that have already done a long travel inside a core network. As another example and in the context of QoS networks, Marco Mellia et Al. [2] have proposed to protect from loss some key identified packets of a TCP connection in order to increase the TCP throughput of a flow over an AF (Assured Forwarding) DiffServ class. In this study, the authors observe that TCP performance suffers significantly in the presence of bursty, non-adaptive crosstraffic or when it operates in the small window regime, i.e., when the congestion window is small. The main argument is that bursty losses, or losses during the small window regime, may cause retransmission timeouts (RTOs) which will result in TCP entering the slowstart phase. As a possible solution, the authors propose qualitative enhancements to protect against loss: the first several packets of the flow in order to allow TCP to safely exit the initial small window regime; several packets after an RTO occurs to make sure that the retransmitted packet is delivered with high probability and that TCP sender exits the small window regime; several packets after receiving three duplicate acknowledgement packets in order to protect the retransmission. This allows to protect against losses, packets that strongly impact on the average TCP throughput. In this paper, we propose to study the prioritisation of certain TCP packets in order to investigate whether we could minimise the transfer delay of short TCP flows without impacting on the long lived connections. Basically, we study how to exploit router functionality to improve the performance of short TCP flows in a best-effort network by giving a higher priority to the first packets of a TCP flow inside a router queue if no others packets belonging to the same flow are already en-queued. One of the main goals of this proposal, called FavourTail, is to investigate and understand the benefits of using a prioritisation forwarding scheme inside a core network. Intuitively, we expect to decrease the transfer delay of small TCP connections but surprisingly, we show that this routing behaviour does not only improve the performance of TCP flows and allows to improve the overall performance in terms of transfer delay when slight congestion occurs. We present the potential benefit of using such solution and analyse the benefit of our scheme over a realistic and complex network topology. We show that the proposed scheme can improve the transfer delay of TCP flows up to $25 \%$.

This paper is organised as follows: the next section II presents some related work; afterwards, section III presents the problem and gives the motivation of this work. Both sections IV and V evaluate through simulations our FavourTail proposal. Finally, section VI concludes and gives the perspectives of this study.

\section{RELATED WORK}

Many research papers have tackled router-based methods to optimise flows transfer. This section presents the most related to our proposal.

In [3], routers memorise the number of bytes of each flow crossing them. Upon reception of a packet, the number of bytes is updated and is added to the queue so that packets in the queue be always sorted based on the number of bytes traversed. The consequence is the flows are given higher priority when they are at the beginning of connection. The drawbacks are routers need flow states, heavy computations are requested to sort the queue, and the number of packets in the flow must be known.

In [4], the authors remove this final condition by computing the number of the packet from the TCP sequence number. To do this, they introduce the following hypothesis: the starting sequence number must have the last $N$ bits $(N=22$ proposed in the article) equal to zero. The drawbacks are TCP senders 
must be modified, the sequence number is more vulnerable to guessing attack and the deployment is difficult: short flows on a standard TCP source will be penalised, since the sequence number of the first packets are misinterpreted by the router as being the $\mathrm{N}^{t h}$ packets. Two queues are used and a threshold. Upon reception of a packet, if the number of bytes of that flow is inferior to a threshold, the packet is enqueued in the priority queue and in the normal queue otherwise. The priority queue is FIFO, while the normal one is sorted by the number of crossing packets. The normal queue is only used when the priority queue is empty.

Another idea is presented in [5]. Only edge routers need flow soft states (soft meaning that they do not need it permanently). They count the packets of each flow and set the DiffServ bits of each packet. Core routers use only DiffServ information. Edge routers mark packets as IN if the current number of packets is inferior to a certain threshold, and OUT if they exceed this threshold.

\section{PRoblem FORMULATION AND MOTIVATION}

The purpose of storing packets at a router queue is to temporarily absorb burst of packets. The idea we want to develop is to prevent short data flows to be enqueued due to a burst induced by long-lived traffic.

To solve this problem, a possible solution is to involve only the end points. Suppose a connection which only needs to send 10 packets. If we assume the sender is aware of this small number of packets to transmit, we could propose to let him choose to send them all in one burst. However, this violates the slow start phase and the congestion control principle. As a matter of fact, it results that a mechanism to favour short flows must necessarily involve routers.

Idea presentation: When a packet arrives at a router, the router first decides whether it is to be rejected or enqueued ${ }^{1}$. If the packet is to be enqueued, the packet scheduling takes place. In the FIFO scheduling policy, the packet is inserted at the top of the queue.

Our packet scheduling proposal, called FavourTail, changes the enqueuing packet process. Indeed, when a packet is enqueued, a check is made in the whole queue to seek another packet from the same flow. If no other packet is found, it becomes a priority packet, otherwise it is added as usual at the top of the queue. Priority packets are added at the beginning of the queue, right after the last priority packet (if there are any). The packet reordering inside a flow is thus avoided.

This scheme is quite similar to a priority queuing scheduling mechanism [7].

The packet scheduling policy proposed in FavourTail can be enabled inside any queue management such as DropTail and RED [1]. In the simulations below, we use DropTail.

Variants: There are many variants of this idea which do not involve flow states on routers. We plan to work on them in

${ }^{1}$ In the case of RED routers [6], packets can be marked instead of being rejected; marked packets belong to enqueued packets in our article. order to understand their effect and drive performances comparison measurements. The following details some possible variants:

- Instead of a binary function (i.e. insert the packet in the priority queue or in the normal one), we could use another function $f(n, m)$ giving the level in the queue where the packet is inserted, where $n$ is the number of packets of the same flow in the queue, and $m$ is the total number of packets in the queue. Function $f$ might be linear, exponential, logarithmic and so on. For example, in classical FIFO, $f(n, m)=m$, i.e. the packet is always added on top of the queue (the difference with Fair Queuing scheduling policy is $f$ also depends on the number of packets of other flows);

- Instead of having only two queues, we could choose to set several ones with number of packets threshold. For example: one which enqueues packets with no other packets belonging to the same flow, another for packets with only few packets (for instance, one or two packets), one for a bit more packets (more than two and less than five) and the last priority queue for all the other packets. However, this solution has to be studied carefully as it might introduce an important overhead;

- Act on dropped/marked packets. When a packet is going to be dropped and if it is a priority packet, then choose the last low priority packet.

Dimensioning: We want to evaluate if FavourTail might have good results in realistic cases. As an example, suppose a flow of $8 \mathrm{Mb} / \mathrm{s}$ (equivalent to $1000 \mathrm{pkts} / \mathrm{s}$ with a 1000 bytes packet size). Suppose also an RTT (Round Trip Time) of 50ms. It results $50 \mathrm{pkts} / \mathrm{RTT}(1000 \mathrm{pkts} / \mathrm{s} * 50 \mathrm{~ms} / \mathrm{RTT})$. If each direction gets half of them, then there are 25 data packets in flight. If there are 10 routers between source and destination, then there are $2.5 \mathrm{pkts} /$ router in average. We therefore consider that there are a few routers where this flow is still prioritised. However, it is more consistent to consider the overall gain obtained by the flow rather than the number of times this flow got a packet with a high priority.

\section{Characteristics:}

- FavourTail does not only favour the beginning of connection but also flows with few packets in flight, i.e. with small congestion window;

- There is no relationship between the routers, i.e. a flow with ten packets in flight, one in each router, is favoured, while another flow with only two packets in flight, both on the same router, is not favoured. This is especially true in the TCP slow start phase: packets are generated in burst, so the probability to have several packets of this flow in a router is higher. A CBR-like (Constant Bit Rate) behaviour, such as the one in TFRC [8], should be much more prioritised, because their packets are distributed across all the routers;

- There are still several cases where a flow with a very small transmission time has the same transmission time in both cases (DropTail and FavourTail): (1) it might 
have only one intermediate router, so the chance to be in concurrence with other flows are smaller, (2) this might be explained because in FavourTail, they never get high priority (it crosses routers which have only high priority packets), (3) it might be because in DropTail the routers are empty (so it is like they would be priority), (4) it might be because on the next router of the next router the prioritisation of the packet does not change anything;

- For priority flows, the more routers are in the path, the greater is the gain in terms of transmission time;

- In terms of security, sources cannot cheat by sending packets at a rate making them always priority, because they cannot estimate how many priority packets are inside routers (a priority packet may be put in the head if there is no other priority packet, but it may be put as number 10 is there are already 9 priority packets in the queue);

- The deployment is in the interest of the person who deploys. Indeed, when a router uses FavourTail, its own users are advantaged: clients receive faster Web pages, and servers reply faster to their Internet clients;

- Starvation may occur for long flows in a router which receives only priority packets. We have not discovered such cases in our simulations, but it may arise in some special cases, such as when new very short flows are created at a regular and small interval, so that a nonpriority packet is blocked by packets from continuously new flows. A solution to avoid starvation is that the normal queue be served from time to time even if the priority queue is not empty; we will tackle this in a future work;

- A solution which acts on two bursty packets exactly like two spaced packets, i.e. which spaces the bursty packets, would avoid TCP problem given below, in section IV-C. We reserve this possible enhancement in a future study.

FavourTail has been implemented in ns2 [9] as an extension of the DropTail queue. The next sections present the results.

\section{Simulation RESUltS ON A SIMPLE NETWORK}

\section{A. Network topology}

We firstly evaluate FavourTail over the simple network topology given figure 1. The links are configured as follows: both access links have a capacity set to $2 \mathrm{Mb} / \mathrm{s}$ while the bottleneck link has a $1 \mathrm{Mb} / \mathrm{s}$ capacity. All links have a transfer delay set to $10 \mathrm{~ms}$. The queue size of routers is 50 (the value by default in ns2). Two FTP/TCP traffics are generated: the first one, $\mathrm{C} 1$, from src1 to dest, and the second one, $\mathrm{C} 2$ (in the second simulation we use TFRC instead of TCP), from src2 to dest. $\mathrm{C} 1$ starts at sec. 0 and ends at sec. 5. C2 starts at sec. 1 and generates only 12 data packets. Obviously, at the beginning of the connection, a SYN packet is generated, and FavourTail will give it a high priority. The tests are made with both policies: DropTail and FavourTail.

\section{B. Results}

TCP: The time elapsed (in seconds) between the last packet sent and the first one for C2 is 0.53 for DropTail and

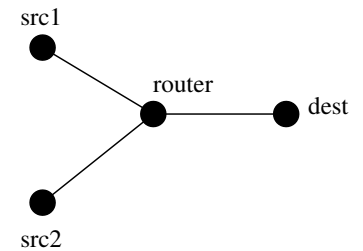

Figure 1. Topology of the simple network.

0.43 for FavourTail. The total number of packets sent by $\mathrm{C} 1$ is 591 in both cases. No packet is lost.

This is a positive result for the user in terms of transfer duration. Indeed, $\mathrm{C} 1$ is not penalised at the end of the connection, while C2 finishes about $20 \%$ sooner. The reason is that the first two packets of $\mathrm{C} 2$ are prioritised by the router. ns 2 reveals that, when arrived at the router, the first packet overtakes 13 slots (packets), while the second one overtakes 14 slots. The analysis can be further developed: As the link transfer time of packets is identical, the difference in time between $\mathrm{C} 1$ and $\mathrm{C} 2$ should correspond to the different processing time of the first two packets by the router; and this time difference $(0.53-0.43=100 \mathrm{~ms})$ should correspond to the processing time of the $13+14=27$ slots (packets) overtaken by the first two packets of $\mathrm{C} 2$.

TFRC: If C2 uses TFRC [8] instead of TCP, the time elapsed between the last packet sent and the first one for $\mathrm{C} 2$ is 0.54 for DropTail and 0.17 for FavourTail. The total number of packets sent by $\mathrm{C} 1$ is 591 in both cases.

This is again a very positive results for the user. $\mathrm{C} 1$ is not penalised at the end of the connection, while $\mathrm{C} 2$ transfer is about 3 times faster. In fact, 6 packets from $\mathrm{C} 2$ are prioritised, gaining each one between 14 and 17 slots.

\section{Discussion}

While the results with this network topology are positive, i.e. the small flow is indeed favoured, the question is why not all its packets are prioritised.

In fact, TCP is a protocol which sends packets in burst. The first burst contains one packet, so it is prioritised. The second burst, sent when the acknowledgement arrives, contains two packets. The first packet is prioritised on the router, but the second one arrives before the first one leaves the router (the link after the router, $10 \mathrm{~ms} / 1 \mathrm{Mb}$, is slower than the link before the router, $10 \mathrm{~ms} / 2 \mathrm{Mb}$ ), hence it is not prioritised. As the queue contains many packets from the long flow, this packet is still in the queue when the packets of the next burst arrive. In fact, because the queue has many packets, none of the following packets are prioritised.

On the other hand, TFRC is a congestion control which sends a smooth (CBR-like) non bursty traffic. More packets than TCP are prioritised, but not all (6 out of 12/13). The reason is that for the seventh packet the throughput of the TFRC flow is a bit higher than the output router link, so this packet arrives at the router before the sixth packet has left. The next packets will experience the same problem. 


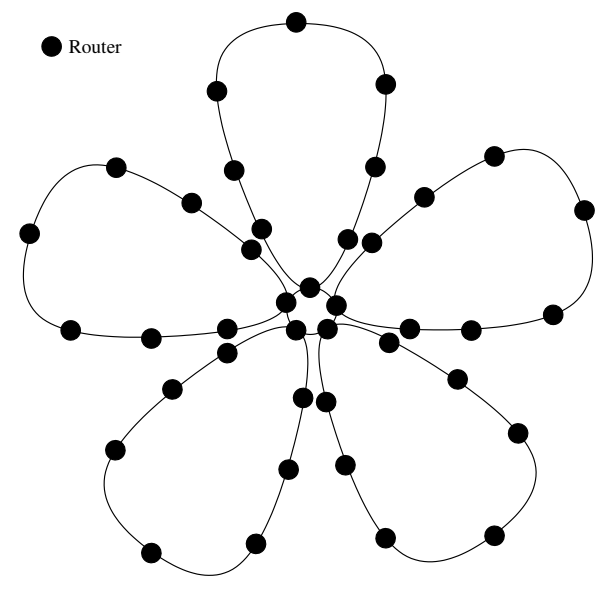

Figure 2. Backbone of the flower network.

\section{Simulation RESUlts ON A COMPLEX NETWORK}

\section{A. Network topology}

In order to evaluate FavourTail over a more realistic case, a more complex topology is used in the next simulations. According to a small study of the xDSL backbone of a Internet provider ${ }^{2}$, most of these networks are built around a central core where several loops are connected. These loops are composed of a small number of routers. The aim of the closed loop is to have a fault tolerance, i.e. when one link becomes unavailable, packets take the other direction so the connection is still assured for all the routers.

For the simulation, a flower with five loops is considered as backbone, each loop has 8 routers, shown in figure 2. Each router (except the 5 core routers) has 2 DSLAMs $^{3}$ connected to it, and each DSLAM has 3 hosts connected to it. Each link has the following characteristics: $10 \mathrm{Mb} / \mathrm{s}$ bandwidth, $10 \mathrm{~ms}$ delay, DropTail (or our FavourTail). The queue size of all the routers is 50 (the value by default in ns2).

We emit 500 FTP over TCP/Newreno connections with random and non identical hosts as source and destination. Each connection starts at a random time between 0 and 20 seconds and sends a random number between 10 and 600 packets.

\section{B. Results}

Several metrics are used to compare DropTail and FavourTail, we divide them in global and short flows specific ones.

Global metrics: Global metrics refer to metrics about the whole simulation. Table I presents some results.

FavourTail globally reduces the transmission time of all flows. As shown in figure 3, the time reduction is globally spread among the flows with higher transmission time. This will be detailed later. As a matter of fact, if a scheduler treats

\footnotetext{
${ }^{2} \mathrm{http} / / /$ support.free.fr/reseau/province.png, not available anymore as of $\mathrm{Au}$ gust 2008. http://www.renater.fr/spip.php?rubrique12 presents the network of another Internet provider; it uses several loops connected to 2 cores.

${ }^{3}$ A DSLAM (Digital Subscriber Line Access Multiplexer) is a network device which connects multiple DSL (Digital Subscriber Line) clients to a high-speed Internet backbone.
}

Table I

GLOBAL METRICS.

\begin{tabular}{|c|c|c|}
\hline & DropTail & FavourTail \\
\hline Sum of transmission times (in seconds) & 2618.68 & 2410.34 \\
Number of lost packets & 2470 & 1608 \\
Number of lost data packets & 913 & 626 \\
\hline
\end{tabular}

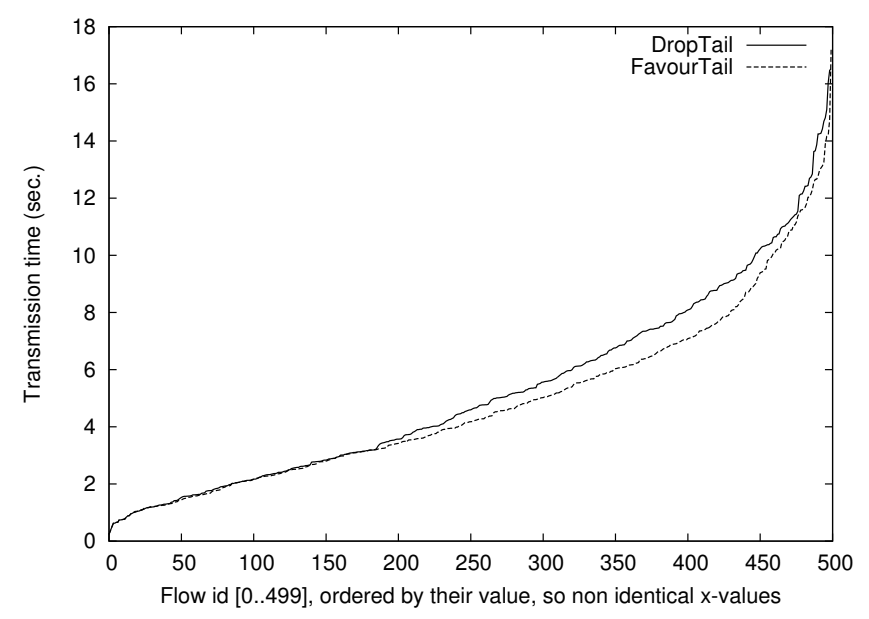

Figure 3. Transmission time of all the flows; the two curves have different values on abscissa.

first shorter tasks, then the total finish time is smaller. This might explain why we obtain a shorter transmission time.

We also notice that the number of lost packets, and of lost data packets, is $30 \%$ smaller with FavourTail.

Short flow specific metrics: Our idea favours packets when they are alone inside the current router (i.e. no other packets from the same flow exist). This leads to the idea that flows which have to send only few packets should be favoured. However, several results prove the contrary.

The transmission time of each flow for both variants is given in figure 4 . They are ordered by the transmission time of the FavourTail variant, in figure 4(a), and the DropTail variant, in figure 4(b). In figure 4(a), it seems that flows with short transmission times are indeed favoured. However, this is not true. This is clearly shown in figure 4(b), where for short transmission times (on the left $\mathrm{x}$-axis in this figure), DropTail variant seems to have smaller times.

It results that using DropTail or FavourTail transmission time is subjective. An objective comparison, i.e. order the abscissa based on the "length" of flow, is therefore needed. Several criteria may be chosen as length of flow: (1) number of packets sent by the flow, (2) number of packets divided by the number of intermediate routers (or links), (3) number of packets divided by the number of concurrent flows inside path routers, and so on. In our context, i.e. favouring packets with few packets, the most appropriate criterion is the number of packets divided by the number of intermediate routers (we use here a simple ratio function for simplicity purpose). A smaller value means more favoured. In fact, the more the packets, the smaller the gain of FavourTail; the more the number of links, 


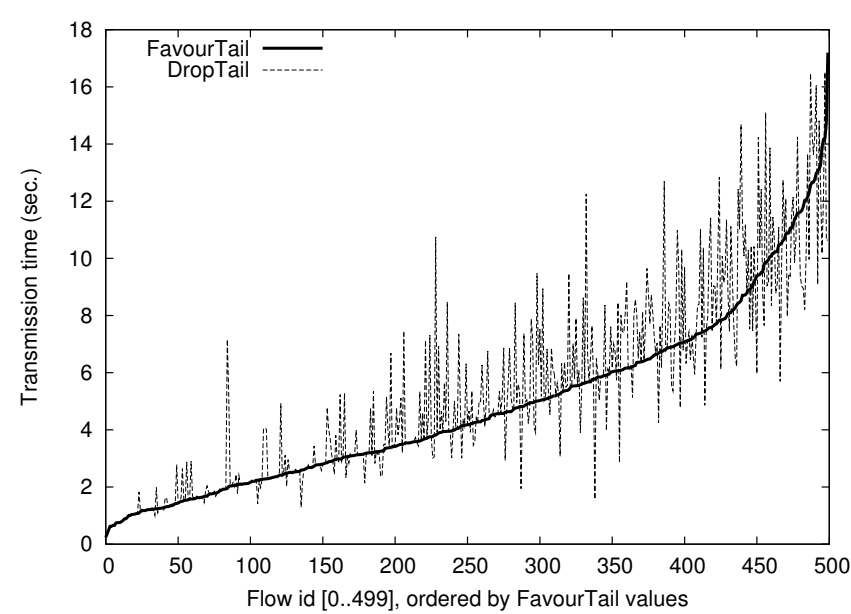

(a) FavourTail

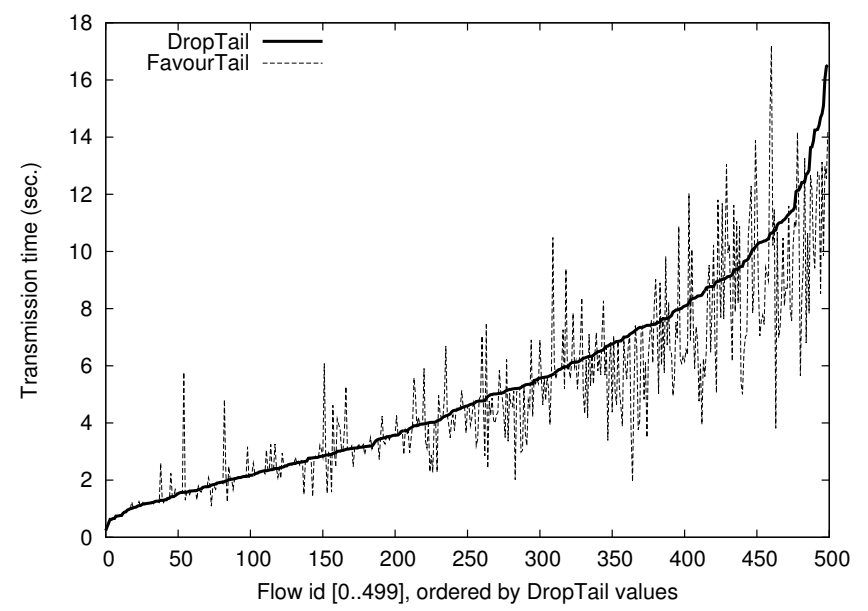

(b) DropTail

Figure 4. Transmission time of all the flows, ordered by the transmission time of FavourTail variant, and DropTail variant.

Table II

STATISTICS OF THE TRANSMISSION TIME OF THE 500 FLOWS, ORDERED BY THE NUMBER OF PACKETS DIVIDED BY THE NUMBER OF FLOW LINKS.

\begin{tabular}{|c|c|c|}
\hline & DropTail & FlavourTail \\
\hline Average & 5.24 & 4.82 \\
Std. Dev. & 3.38 & 3.06 \\
Min & 0.24 & 0.24 \\
Max & 16.52 & 17.2 \\
\hline
\end{tabular}

the higher the gain of FavourTail.

Table II presents the statistics of the transmission time ordered by the number of packets divided by the number of links. We can observe a slight advantage for FavourTail. The statistics ordered by the number of packets show similar results (albeit the difference between both variants is a bit further reduced).

Finally, another round of simulations have been done, where several parameters of the simulation have been individually changed, and each time the results are quite similar. The

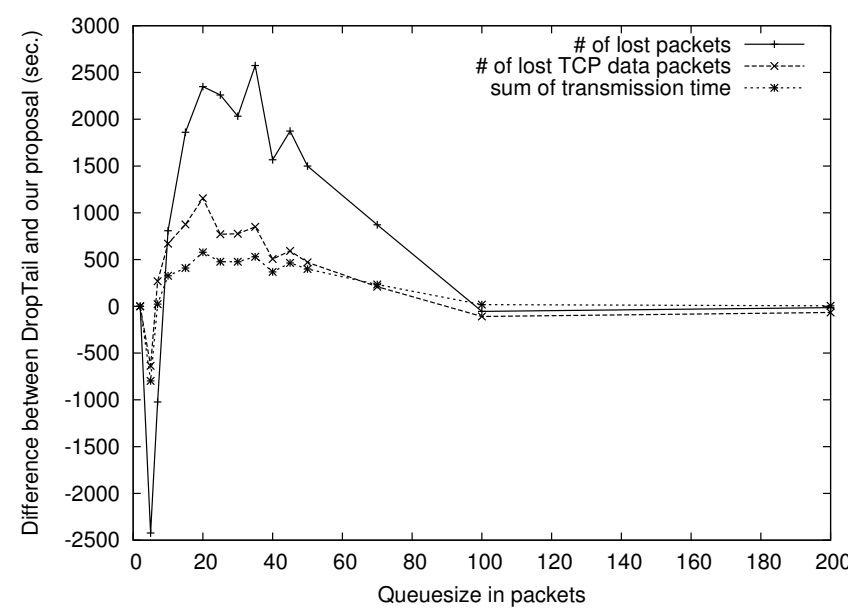

Figure 5. Comparison between DropTail and our proposal when varying the queue size.

parameters changed are:

- all the traffic is TFRC instead of TCP;

- the data size of flows follows a Pareto distribution with an average of 300 packets and a shape of 1.2;

- the first 50 flows send between 1 and 10 packets and all the others send between 200 and 800 packets;

- each core router has attached only one DSLAM, and each DSLAM has only one host attached.

We plan to further investigate these simulations, as given in future work.

Experiments with variable queue buffer size: In this experiment, we vary the queue size of each router buffer from 2 to 200 packets. This allows to emulate a congestion level inside the whole core network. We use the flower network topology previously presented with random size of data transfer (but with the following difference which however gives similar results: the first 50 flows send a number of packets ranging from 1 to 10 packets and all others send between 200 and 800 packets). Then, we report the number of dropped packets and the number of TCP data packets and the sum of transmission time of all data transfer during the experiment. These metrics are those already presented at the beginning of this section.

In order to verify the benefit introduced by our solution at a macroscopic level, we show in figure 5 the difference between the results obtained by DropTail and our proposal. When this difference is positive, our proposal gets better performance than DropTail. As expected, and in order to verify our implementation, when the queue size is very small (i.e. when the queue size is set to two packets), the difference is nil meaning there is no advantage for both mechanisms. When the queue size is very large (i.e. higher than hundred packets), the performance realized by both mechanisms is quite similar. The fixed TCP window size of all sources explains this later result: the buffer occupancy of each router queue is stabilized since the emitted traffic is not growing anymore due to this fixed size. This result allows to stand that when buffers size 
are large, meaning there is no congestion inside the network, our proposal does not bring any advantage. However, when the queue size is ranging from 10 to 70 , that can symbolize a congestion level considered as severe to slight, our proposal allows to decrease the number of dropped packets and as a consequence, the number of retransmitted packets which directly results by a lower transmission time for the TCP flows.

At a microscopic level, the results obtained by all flows are similar to those presented in figure 3. Despite the fact that the overall performance gets better, we cannot see a lower transfer time for short TCP flows as illustrated in figures 6 for various queue sizes.

\section{CONCLUSION}

In this paper, we have presented and evaluated a novel forwarding scheme. We show that this scheme leads to interesting properties allowing to decrease the overall transfer delay of TCP flows in the context of best-effort networks. The results obtained are quite unexpected, as intuitively we would expect a stronger benefit of this mechanism to short TCP flows and on the contrary, measurements show an overall benefit for long flows without high impact over short ones with our proposal compared to DropTail. Indeed, the main findings are that FavourTail decreases the number of packets dropped and as a primary consequence, the sum of transmission times is thus reduced. However, the short flows are not noticeably favoured compared to the other longer flows.

In a future work, we aim at investigating, through a larger measurements campaign, this forwarding scheme and in particular with other transport protocols such as TFRC in order to verify whether we would benefit similar properties.

\section{ACKNOWLEDGEMENTS}

Authors thank Pascal Anelli, François Spies for their remarks about packet prioritisation, and anonymous reviewer.

\section{REFERENCES}

[1] J. F. Kurose and K. W. Ross, Computer Networking, 4th ed. AddisonWesley, 2008.

[2] M. Mellia, I. Stoica, and H. Zhang, "Tcp-aware packet marking in networks with diffserv support," Comput. Netw., vol. 42, no. 1, pp. 81100, 2003.

[3] I. A. Rai, E. W. Biersack, and G. Urvoy-Keller, "Size-based scheduling to improve the performance of short TCP flows," IEEE Networking, vol. 19, no. 1, pp. 12-17, Jan.-Feb. 2005.

[4] K. Avrachenkov, U. Ayesta, P. Brown, and E. Nyberg, "Differentiation between short and long TCP flows: predictability of the response time," in INFOCOM, ser. 7, vol. 2. Hong Kong: IEEE, Mar. 2004, pp. 762-773.

[5] X. Chen and J. Heidemann, "Preferential treatment for short flows to reduce web latency," Computer Networks, vol. 41, no. 6, pp. 779-794, Apr. 2003.

[6] S. Floyd and V. Jacobson, "Random early detection gateways for congestion avoidance," IEEE/ACM Transactions on Networking, vol. 1, no. 4, pp. 397-413, Aug. 1993.

[7] D. Zhang, H.; Ferrari, "Rate-controlled static-priority queueing," in Proceedings of IEEE Infocom, Mar. 1993.

[8] S. Floyd, M. Handley, J. Padhye, and J. Widmer, "TCP Friendly Rate Control (TFRC): Protocol specification," Sep. 2008, RFC 5348.

[9] "Network simulator — ns-2," http://www.isi.edu/nsnam/ns/.

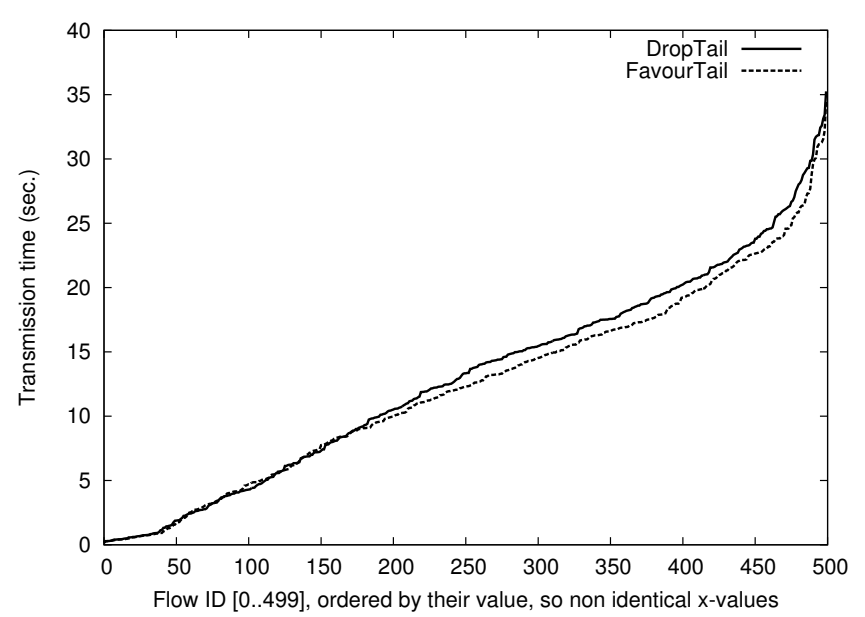

(a) queuesize $=10$

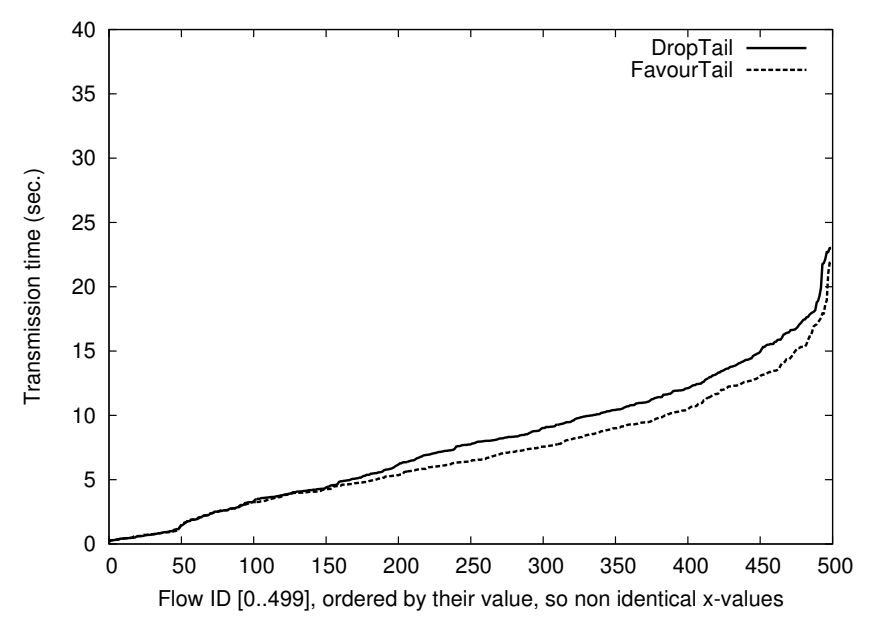

(b) queuesize $=30$

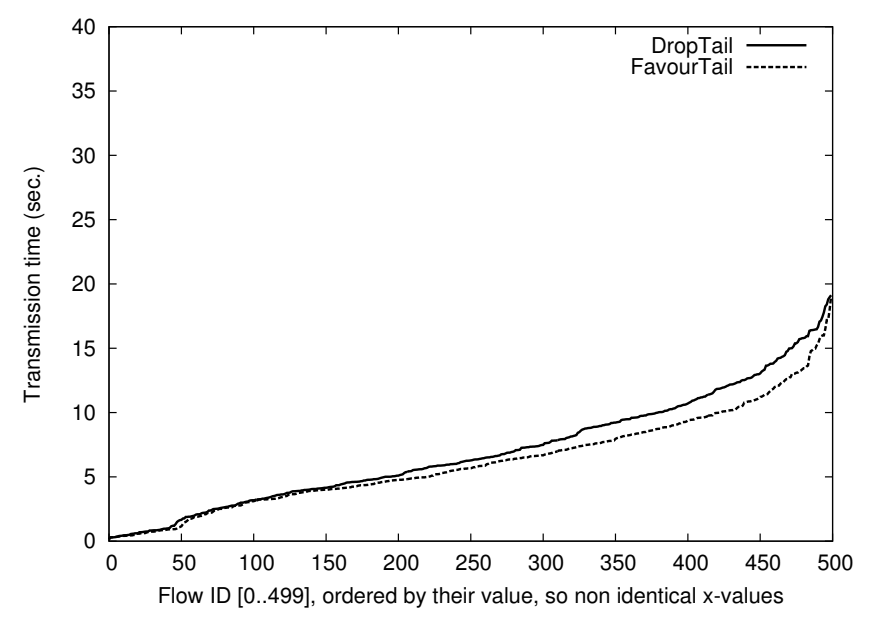

(c) queuesize $=50$

Figure 6. Transmission time of all flows as a function of the queue size. 